\title{
Beziehungsweisen jenseits der Zweisamkeits(ver)ord- nung oder: Zur Produktion der Grenze: Wer mehr liebt, hat UnRecht ${ }^{1}$
}

$\mathrm{Zu}$ den in jüngster Zeit erschlossenen und kritisch debattierten Domänen der Rechtstheorie gehören die Verankerung des Heterosexismus im Recht ${ }^{2}$ sowie die Aufdeckung der damit einhergehenden Diskriminierung der Zweigeschlechtlichkeit. ${ }^{3}$ Diese Errungenschaften queer-feministischer Rechtstheorie grenzen an einen noch unberührten Kontinent. Dieser betrifft die Beziehungsweisen der miteinander in Beziehung stehenden Individuen, welche sich in Subjektivationsprozessen niederschlagen und im Recht gleichzeitig objektiviert wie prozessiert werden. Die in der westlichen Gesellschaft dominierende Beziehungsweise kreist wesentlich um die Sonne exklusiver Zweisamkeit und wird als Beziehungsform an und für sich nicht in Frage gestellt. Nur zwei Personen können sich demnach >wirklich und in romantisch-zärtlicher Zuwendung lieben, nur zwei Personen können daher eine sinnvolle und stabile, ungefährdete und ungefährliche Lebens- und Wirtschaftsgemeinschaft bilden, und nur zwei Personen sollten im Idealfall in dieser Zweisamkeit gemeinsam (ihre) Kinder großziehen. Dass ein solches Beziehungsmodell genauso wenig >natürlich ist wie Heterosexualität (worüber mittlerweile ein leidlicher Konsens herrscht) oder Zweigeschlechtlichkeit (eine prekäre Position, die derzeit sehr umkämpft ist, aber zweifelsohne in den Rechtsdiskurs eingebracht wurde), wird im (deutschsprachigen) juristischen Kontext bislang nicht diskutiert.

Ein wenig Licht in dieses streng strukturierte Beziehungsgeflecht zu bringen, beansprucht der vorliegende Beitrag. Dabei wird besonders die Frage in den Blick genommen, wie Recht ganz bestimmte Beziehungsweisen produktiv macht $\mathrm{zu}$ Ungunsten von vorstell- und lebbaren Alternativen.4 Hierbei richte ich den Fokus sowohl auf die Beziehungsformen wie gleichzeitig auch auf die Rechtsform, in der soziale Verhältnisse als rechtlich tragfähige Beziebungsweisen verhandelt werden. Gerade die Form der gesetzlichen Regulation der Intimität ist bislang im Kontext von Rechtstheorie nicht hinlänglich berücksichtigt worden.' Diese Form, begriffen als »eigene Dichte« des Rechts, führt zu effektiven Verselbständigungsprozessen, innerhalb derer Soziales naturalisiert, verdinglicht und in einzelnen Individuen und Rechtssubjekten vereinzelt wird, um den Menschen dann wiederum quasi objektiv - fetischisiert - im Recht als Tat-Sache entgegenzutreten und darüber schließlich gesellschaftliche Zusammenhänge zu stiften. ${ }^{6}$

\footnotetext{
I Für konstruktive sowie praktische Kritik danke ich Dietmar Flucke.

2 Vgl. Elisabeth Holzleithner, Grenzziehungen. Pornographie, Recht und Moral, Wien 2000.

3 Vgl. Adrian de Silva (in diesem Heft).

4 Vgl. Michel Foucault, Sexualität und Wahrheit Bd.I, Der Wille zum Wissen, Frankfurt am Main I979, I I4.

5 Vgl. Jean L. Cohen, Personal Autonomy and the Law: Sexual Harassment and the Dilemma of Regulating >Intimacy<, Constellations, 6/4 (1999), 443-472, 445.

6 Vgl. Sonja Buckel, Subjektivierung und Kohäsion. Zur Rekonstruktion einer materialistischen Theorie des Rechts, Weilerswist 2007.
} 
Der Sorgerechtsstreit einer jungen Mutter 1999 in Memphis, Tennessee, zeigt in außergewöhnlicher Klarheit die Problematik und (juristischen) Konsequenzen der regulativen $Z$ weierbeziehungsnorm.

April Divilbiss wurde im November 1998 ihre zu dem Zeitpunkt dreijährige Tochter Alana Moore entzogen, weil sie mit ihrem Ehemann Shane Divilbiss und ihrem weiteren Lebenspartner Chris Littrell (keiner der beiden war biologischer Vater des Kindes) in einer polyamourösen Lebensgemeinschaft lebte.

Nachdem der biologische Vater des Kindes i 995 schnell verschwunden war, sobald er von Aprils Schwangerschaft hörte, heirateten April und Shane Divilbiss kurz nach der Geburt von Tochter Alana I996. Im Januar I 997 verliebten sich April Divilbiss und der Trauzeuge und Freund des Paares, Chris Littrell. Der aus dieser neuen Konstellation erwachsende Konflikt wurde nicht traditionell - durch Austausch eines Partners - gelöst; vielmehr beschlossen alle drei gemeinsam, ab März 1997 in einer polyamourösen Triade zusammenzuleben. Auslöser für die Klage der Großmutter Donna Olswig - der Mutter des abwesenden leiblichen Vaters des Kindes - war eine MTV-Dokumentation über polyamouröse Lebensweisen, in der über die junge Familie berichtet wurde. ${ }^{8}$ Ein Richter ließ das Kind im November nach einer Sichtung des MTV-Videos umgehend aus der Familie entfernen und ignorierte - so reklamierte Divilbiss' Anwalt Asa Hoke - die staatlich garantierten Rechte der Mutter auf ein ordentliches Gerichtsverfahren. ${ }^{9}$ Der vielbeachtete Fall erfuhr breite Unterstützung nicht zuletzt in Form einer Fundraising-Kampagne des Loving More Magazin ${ }^{10}$ - es wurde auf einen >Stonewall ${ }^{\text {II }}$ vergleichbaren Ausgang gehofft. Der Prozess fand schließlich am r6. April 1999 im Juvenile Court of Memphis statt.

Die in diesem Fall angelegte Dramatik eröffnet das Feld der Beziehungsweisen aus der ungewohnten Perspektive alternativer Beziehungsformen, deren einvernehmlich geregelte Alltags- und Beziehungspraxis durch die gesetzliche Regulierung der Intimität unterworfen werden.

\section{Polybegehren-Herausforderung der Beziehungs(ver)ordnung?}

Das Prinzip der >romantischen Zweierbeziehung - kurz: >RZB ${ }^{12}$ - ist einfach und wird in klassischer Literatur wie in den Reflexen der Kulturindustrie so intensiv wie einseitig thematisiert: Drei sind eine_r zuviel. So verhandeln die

7 In the Matter of A.M., No. Ki7r 9 (Juv. Ct., Memphis and Shelby County, Tenn., Apr. I6, I999).

8 Sex in the '9os: It's a Group Thing; Vgl. John Cloud, Henry \& Mary \& Janet \& .... Is Your Marriage a Little Dull?: The >Polyamorists< Say There's Another Way, Time Magazine Nov. Is I 54/20 (I 999).

9 Vgl. Respondent Natural Mother's Motions for an Immediate Hearing (Fn. 7).

Io Das Loving More Magazin ist eine vierteljährlich erscheinende polyamouröse Zeitschrift sowie eines der Organisationszentren und Sprachrohre der US-amerikanischen Polyamory-Bewegung, auf die im Folgenden eingegangen wird (www.lovemore.com).

I I Die sStonewall-Unruhen beziehen sich auf eine Serie von gewalttätigen Konflikten zwischen Lesben/ Schwulen und Polizeibeamten in New York City, innerhalb derer sich im >Stonewall Inn erstmalig eine signifikant große Anzahl von Gästen einer Verhaftung im Rahmen einer Razzia in der Nacht vom 27. zum 28.6.1969 widersetzte, was als Wendepunkt in der amerikanischen Lesben- und Schwulenszene im Kampf für Gleichbehandlung und Anerkennung gilt. Vgl. David Carter, Stonewall: The Riots That Sparked The Gay Revolution, New York 2004.

I 2 Fremdgenese, Glück im Unglück. Un/Möglichkeiten der Romantischen Zweierbeziehung, in: A.G. Gender-Killer (Hrsg.), Das Gute Leben. Linke Perspektiven auf einen besseren Alltag, Münster 2007, 95-I I 3 , 95 . 
Briest, des jungen Werther, sowie das Beziehungs-Experiment in Goethes Wablverwandtschaften das außereheliche - und damit über Zweisamkeit hinausgehende - Begehren in salonfähiger Tragik und lassen dabei wenig Zweifel über die auf dem Fuße folgende Katastrophe. MTV hingegen wirbt in noch klareren Worten für ein $200 \mathrm{I}$ in den USA angelaufenes Reality-Format: »Die drei-isteiner-zu-viel-Dating-Show: >DisMissed < ist die Show über dich, die Person deiner Träume - und deinen Nebenbuhler ${ }^{.}{ }^{13}$

Diese kulturelle Form der RZB ist vielfach kritisiert ${ }^{\mathrm{I} 4}$ und erkannt worden als den Menschen gerade nicht innewohnende >Natur<, sondern als eine spezifisch geformte Begehrenskonstellation, welche einer bestimmten gesellschaftlichen Situation entspricht. ${ }^{\text {Is }}$ Analysen der RZB müssen sich daher den Konstitutionsbedingungen des zweisamen Begehrens zuwenden, welches bereits seit zwei Jahrhunderten formale Kontinuität beweist. ${ }^{16}$ Die Analysen Luce Irigarays von Weiblichkeit, Subjektivität und Begehren haben philosophiegeschichtliche Kontinuitäten als Stützen der permanenten Reproduktion heterosexueller Beziehungsmuster ausgewiesen; ${ }^{17}$ Judith Butler hat daran anschließend in ihren Arbeiten zur Dekonstruktion der Zweigeschlechtlichkeit bereits evidente Hinweise für ein darin aufgehobenes Begehren bestimmter Beziehungsweisen geliefert. ${ }^{18}$ Im Anschluss an Alfred Lorenzer lässt sich in der kritischen Analyse des auf exklusive Zweisamkeit gerichteten Begehrens noch einen Schritt weiter gehen: Lorenzers Konzeption der Interaktionsform als geronnener Erinnerungsspur sozialen Erlebens als körperliches ermöglicht, Vorstellungen von einer körperlichen Dinghaftigkeit aufzulösen, ${ }^{19}$ und darüber das lebensgeschichtliche Gewordensein körperlichen Begehrens zu dechiffrieren. Gesellschaftlichkeit wird über primäre Bezugspersonen bereits vor der Geburt durch die Art ihres Interagierens an den »immer schon sozial exponiert[en] « ${ }^{20}$ Fötus herangetragen und strukturiert die vorhandenen >Natur-Möglichkeiten<. ${ }^{21}$ Dieser Prozess setzt sich nach der Geburt fort, wobei die Interaktionen grundsätzlich als Aushandlungsprozesse zu verstehen sind; wenngleich die Dominanz der primären Bezugspersonen in den Interaktionen unübersehbar ist, entwickelt sich die Eigenständigkeit des Kindes in dem Maße, in dem es seine Erlebnisse zu strukturieren, $\mathrm{zu}$ symbolisieren und abzugrenzen versteht. ${ }^{22}$ Die einzelnen Bedürfnisse formieren sich so in dialektischer Auseinandersetzung mit innerer und äußerer Natur zu einem körperlich manifestierten Begehren. Dass in diesen

I 3 Http://www.mtv.de/tv/shows/225243, letzter Zugriff am 30.5.2008; in der Show buhlen zwei junge Frauen um einen jungen Mann (und vice versa), der dann am Ende des Spektakels eine der beiden abserviert.

I4 Einen Überblick über und eine umfassende Textsammlung zur Kritik an und Debatten um die romantische Zweierbeziehung auf: http://liebe.arranca.de, letzter Zugriff am I 3.6.2008.

Is Vgl. Julia König, Begehrtes Ich. Zum Verhältnis begehrlicher Beziehungsweisen, diskus 55/200I (2006), 20-28. I6 Das ausgeprägte Affären-Wesen der bürgerlichen Gesellschaft bestätigt in seiner konstitutiven Heimlichkeit und Nicht-Gesellschaftsfähigkeit nur die Exklusivität der Zweierbeziehungen, wobei sie gleichzeitig individuell ein der Zweierbeziehungsmoral implizites Potenzial zum Unterlaufen traditioneller sexueller Hierarchien und Ordnungen aufweisen; vgl. Petula Sik Ying Ho, The (Charmed) Circle Game: Reflections on Sexual Hierarchy Through Multiple Sexual Relationships, Sexualities 9/5 (2006), 547-564.

I7 Vgl. Luce Irigaray, Speculum. Spiegel des anderen Geschlechts, Frankfurt am Main I980.

I 8 Vgl. Judith Butler, Das Unbehagen der Geschlechter, Frankfurt am Main I 99I.

I9 Alfred Lorenzer, Die Sozialität der Natur und die Natürlichkeit des Sozialen. Zur Interpretation der psychoanalytischen Erfahrung jenseits von Biologismus und Soziologismus. Ein Gespräch zwischen Alfred Lorenzer und Bernard Görlich, in: Bernard Görlich/Alfred Lorenzer/Alfred Schmidt (Hrsg.), Der Stachel Freud. Beiträge und Dokumente zur Kulturismus-Kritik, Frankfurt am Main I980, 297-349, 34I. 20 Lorenzer (Fn. 19), 344.

2I Alfred Lorenzer, Trieb und Gesellschaft, in: Ulrike Prokop (Hrsg.), Die Sprache, der Sinn, das Unbewußte. Psychoanalytisches Grundverständnis der Neurowissenschaften, Stuttgart 2002, I I9-I 34, I 3 I.

22 Vgl. Alfred Lorenzer, Zur Begründung einer materialistischen Sozialisationstheorie, Frankfurt am Main I 972. 
Prozessen auch bestimmte - allgemein gesellschaftsspezifische sowie durch die individuelle, lebensgeschichtliche Aneignung besondere - Beziehungsweisen vermittelt werden, die in die körperlich-seelische Struktur der Einzelnen eingegangen sind, ist somit wenig überraschend.

Diverse widerständige Praktiken formulieren die Kritik an der romantischexklusiven Zweisamkeit auf unterschiedliche Weise alltagspraktisch aus.

Kritiker_innen schlagen $>$ falsches Zitieren ${ }^{23}$ von RZBs vor: Romantisierung von Freund_innenschaften, Wahlfamilien, weniger exklusive Privilegien für die Zweierbeziehung. ${ }^{24}$ Bini Adamczak fordert »Kollektivität auf erweiterter Stufenleiter, nicht als umfassender Kreis also, eher als Netz, Vielheit ineinander geschobener, proklamiert offener Ringe «, ${ }^{25}$ die Bewegungen auf der Ebene der Subjektivität erwirken und - in utopischer Perspektive - gesellschaftliche Umwälzungen nach sich ziehen.

Praktische Kritik der Zweierbeziehungsweisen ist in den USA unter dem Namen >Polyamory< organisiert. ${ }^{26}>$ Polyamory< lässt sich als ein Sammelbegriff für vielfältige nicht-monogame Beziehungsentwürfe verstehen, die unter diesem Namen etwa seit den I 990er Jahren vornehmlich in den USA einige Verbreitung gefunden haben. ${ }^{27}$ Die lebenspraktischen Ausformungen dieser Beziehungen sind so vielfältig wie veränderlich; trotz den durch die größere Offenheit fluide(re)n Strukturen wird jedoch grundsätzlich betont, dass es um verantwortliche Nicht-Monogamie ${ }^{28}$ geht, die zum Teil vehement abgegrenzt wird von dagegen als >unverantwortlich überhaupt. ${ }^{29}$ Das Emanzipative der Bewegung leidet unter solchen - unnötigen - Einschränkungen, die auf die ambivalente Verortung von >Polyamory< in unterschiedlichen gesellschaftlichen Diskursen um Sexualität verweisen: Indem Polyamory über Liebe und Intimität definiert wird und diese Gefühle zum Inbegriff von Verantwortung stilisiert werden, verstärkt Polyamory den normativen Gehalt dieser Beziehungsideologien. Gleichzeitig wird die bestehende Kultur der $>$ Zwangsmonogamie $33^{\circ}$ herausgefordert. Die Betonung der $>$ Verantwortlichkeit ist hier jedoch auch im Kontext der Reflexion (oft geschlechtsspezifisch) unterschiedlicher Verletzungsoffenheiten $\mathrm{zu}$ verstehen, die sich in kollektiven Beziehungserfahrungen niedergeschlagen haben.

Im Gegensatz zu vergleichbaren sozialen Bewegungen des 20./2 I. Jahrhunderts wie der feministischen oder der LGBT-Bewegung, mit denen polyamouröse Aktivist_innen sich explizit solidarisch zeigen, zeichnet sich bisher in der Poly-

23 Im Sinne Judith Butlers; vgl. Judith Butler, Körper von Gewicht, Frankfurt am Main I 993.

24 Fremdgenese (Fn. I 2), 107.

25 Bini Adamczak, Theorie der polysexuellen Ökonomie, diskus 55/2006, I 2-19, I 9.

26 Vergleichbare Szenen entwickeln sich mit einigen Jahrzehnten Verzögerung in europäischen Großstädten; im deutschsprachigen Raum in Berlin: http://poly-berlin.de; Luzern: http://www.polyamory.ch; Wien: http://www.polyamory.at; Rechtsstreitigkeiten sind hier jedoch (noch) nicht virulent. Als deutschsprachige Publikationen sind zu nennen: Bärbel Schlender/Erhard Söhner (Hrsg.), Ein Frühstück zu Dritt - Leben und Lieben in Mehrfachbeziehungen, Wien 2006; Laura Méritt/Traude Bührmann/ Nadja Boris Schefzig (Hrsg.), Mehr als eine Liebe - Polyamouröse Beziehungen, Berlin 2005 .

27 Der Begriff >Polyamory< wird zumeist auf einen Artikel von Morning Glory Zell (I990) zurückgeführt. Die bereits in den I970er Jahren diskutierten Beziehungskonzepte werden unter diesem Begriff erst seit den I 990er Jahren gefasst, der erst in jüngster Zeit Eingang ins Oxford English Dictionary gefunden hat. Vgl. Melita Noël, Progressive Polyamory: Considering Issues of Diversity, Sexualities 9/5 (2006), 602-620.

28 Vgl. Dossie Easton/Catherine A. Liszt, The Ethical Slut. A Guide to Infinite Sexual Possibilities, San Francisco 1997; Marcia Munson/Judith P. Stelboum (ed.), The Lesbian Polyamory Reader, New York I999; Ryam Nearing, Loving More: The Polyfidelity Primer, Hawai 1992.

29 Analyse und Kritik in: Christian Klesse, Polyamory and its >Others $<$ Contesting the Terms of Non-Monogamy, Sexualities 9/5 (2006), 565-583.

30 Elizabeth F. Emens, Monogamy's Law: Complusory Monogamy and Polyamorous Existence, New York Review of Law \& Social Change 29/277 (2004), 277-376. 
amory-Szene kein vergleichbarer Kampf um eigene Rechte oder gesetzliche

Anerkennung ihrer Beziehungsformen ab, wie Hadar Aviram exemplarisch in einer qualitativen Studie in der San Francisco Bay Area (2008) zeigen konnte. ${ }^{3 \mathrm{I}}$ Vielmehr zeichnet sich in der Szene große Skepsis gegenüber dem Recht als sinnvollem Instrument im Kampf um die praktizierte Lebensweise ab; GruppenHeirat als vorstellbares Konzept der Anerkennung der Beziehungen ist kein erklärtes Ziel der Bewegung. ${ }^{32}$

\section{Juridische Normalisierung - It is just that simple.«33}

Die romantische Illusion der ewigen Liebe mit den damit verbundenen exklusiven nur in dieser einen Liebesbeziehung zu befriedigenden Gefühlen, die jede Zweierbeziehung chronisch überlasten und eine mehr oder weniger abwechslungsreiche serielle Monogamie erwirken, findet im Recht seine Entsprechung, Manifestation und formgebende Praxis zugleich: Wer sich mit wem versichern kann, welche steuerlichen Vergünstigungen es für wen (nicht) gibt, wer was (ohne Erbschaftsteuer) erbt und wer zu welchen Kindern in welcher Beziehung steht, sind Fragen, die nicht den Alltagshandlungen der Einzelnen überlassen werden, sondern die in juridischen Verfahren codiert werden. Denn Gerichtsprozesse, Gesetzgebungs- oder Verwaltungsverfahren »interpretieren nach bestimmten Regeln die soziale Wirklichkeit als rechtliche [...]. Die einzelnen AkteurInnen sind nicht mehr in der Lage, auf ihre eigenen Verhältnisse zuzugreifen, wenn sie erst einmal in diese Verfahren Eingang gefunden haben «.34 Das gilt genauso für den Teil der Rechtskommunikationen, die außerhalb von juridischen Verfahren stattfinden und dennoch vor dem materiellen Hintergrund, immer gerichtsförmigen Verfahren zugänglich zu sein.

Das in den $\$ \iint_{\text {I }} 297$ bis I 588 BGB formulierte Eherecht lässt sich mit Antonio Gramsci als hegemoniales Recht verstehen, als Ergebnis eines gesellschaftlichen Organisationsprozesses, in dem bestimmte Zweier-Beziehungsweisen als hegemoniale rechtlich codiert werden. Über soziale Kämpfe findet die im Recht ausgearbeitete Weltanschauung indes Eingang in die Selbstkonzeptionen der Einzelnen: Das im Eherecht manifestierte Ideal der romantischen Zweierbeziehung lässt sich demnach als gesellschaftliche Konstruktion oder Norm begreifen, welche tief in den Gewohnheiten der Lebensführung der Einzelnen Wirkungsmacht entfaltet ${ }^{35}$ und ihr Begehren nach Zweisamkeit mit konstituiert, wie sich mit Lorenzer ${ }^{6}{ }^{6}$ differenziert zeigen lässt.

Die Verhandlung des Sorgerechtsfalls Alana Moore im April I999 am Juvenile Court of Memphis verlief unter den Vorzeichen des Zweisamkeitsdogmas.

Zunächst stellte der Anwalt der Großmutter den Antrag, ein vom Gericht selbst in Auftrag gegebenes Expert_innen-Gutachten über den Zustand der Dreijährigen und mögliche negative Einflüsse durch die Lebensweise ihrer drei Eltern aus

3I Hadar Aviram, Make Love, Not Law, Journal of Bisexuality, Vol. 7/3-4 (2008), im Erscheinen, I-33.

32 Diskutiert wird polyamouröse Gruppen-Heirat durchaus - allerdings von hysterischen Konservativen wie Senator Rick Sanatorum oder Stanley Kurtz, die in einer Legalisierung der Homo-Ehe ein Einfallstor (»slippery-slope« argument) für Gruppenheirat, Inzest, und einen moralischen Zusammenbruch der USamerikanischen Gesellschaft wittern. Vgl. Edward Ashbee, Polyamory, Social Conservatism and the Same-Sex Marriage Debate in the US, Politics 27/2 (2007), I0I-I07.

33 Honorable Herbert Lane, Transcript (Fn. 7).

34 Buckel (Fn. 6), 240.

35 Vgl. dazu Alex Demirović, Der nonkonformistische Intellektuelle. Die Entwicklung der Kritischen Theorie zur Frankfurter Schule, Frankfurt am Main 1999.

36 Vgl. Lorenzer (Fn. I9). 
der Beweisaufnahme auszuschließen, da er keine Zeit gehabt habe, eigene Expert_innen heranzuziehen. Richter Herbert Lane folgte der Argumentation und setzte durch, dass die positiven Befunde der Expert_innengruppe - dem Mädchen fehle nichts, es scheine ihr sehr gut zu gehen - keinen Eingang ins Verfahren fanden. Divilbiss' Anwalt Hoke hatte hingegen seinen Antrag auf Zurückführung der Tochter zu ihrer Mutter wesentlich auf dem Gutachten aufgebaut. 37 Davon zeigte sich der Richter jedoch unbeeindruckt und lehnte den Antrag ab, ohne ihn verlesen zu lassen. Nachdem auch er sich das Video von MTV angesehen hatte, verzichtete er auch auf die Aussage der Mutter April Divilbiss und stellte in seiner prompten Urteilsverkündung fest:

"What I have got here is a young lady who has decided to have an alternative life style and the issue becomes is that life style, in fact, detrimental to this child. You know, parents oftentimes are called upon to make great sacrifices for their children. And when she was faced with that, when some guy came to her and said I' $m$ in love with you too although you are married, you know, most people would have said, well, hey, I'm married; forget it. But, no, she decides, well, why not. I'll just - I'll have both of them. I can have my cake and eat it too. Well, parents can't do that. Parents have to set the correct examples for the kids. And part of the statute says that you have to be concerned with the moral upbringing of the child. [...] So here is how we are going to resolve this, folks: Custody of the child is going to be placed with the Department of Children's Services with an extended visit in the grandmother's home until such time as the mother resolves her situation. Now I am not about to put that child back into a situation where all three of these people are in the same bed. She has got a legal husband. Make a choice. It is just that simple. «38

$\mathrm{Da}$ er in diesem Sorgerechtsstreit im besten Interesse des Kindes entscheiden müsse, könne er nicht warten, bis Alana durch die Fragen ihrer Spielkamerad_innen zu der alternativen Familienkonstellation oder gar durch den Anblick ihrer drei Eltern gemeinsam im Bett39 »traumatisiert « würde und müsse jetzt handeln.

Wenn sich Gerichtsentscheide als »verdinglichte Formen menschlicher Sozialität « 40 begreifen lassen, so lässt sich die Monstrosität des Divilbiss-Falls in zweierlei Hinsicht dechiffrieren:

Abgesehen von dem offenbar moralisch ungemütlichen Klima in Memphis, Tennessee, tritt erstens deutlich die produktive Präsenz des Politischen im Recht hervor. So lässt sich die Argumentation des Richters Lane als eine machtbasierte Entscheidung verstehen, die jedoch auch hier über spezifisch-juristische Argumentationsfiguren getroffen werden muss. Damit ist sie keinesfalls willkürlich: Denn auch sie folgt gleichermaßen dogmatischen Anforderungen der Rechtsform wie einer universalisierbaren Argumentation. . $^{\text {I }}$ Richter Lane bezieht sich auf eine bestimmte Tradition der Rechtsprechung zum »besten Wohl des Kindes«, die er jedoch im Hinblick auf die hegemoniale Einschätzung der NichtMonogamie in besagter juristisch-spezifischer Argumentationsweise auszulegen versteht. Damit agierte er im Rahmen hegemonialer Diskurse, innerhalb derer er sein Urteil entwickelt.

Zweitens verweisen sowohl die pädagogisch-therapeutischen Empfehlungen des (ungehörten) gerichtlichen Expert_innengremiums ${ }^{42}$ wie auch das Urteil des Gerichts auf den erzieherischen und kreativen, produktiv-bildenden Charakter

37 Motions filed with the judge alleging I I violations of constitutional rights which was rejected (without being read) (Dokument liegt der Autorin vor).

38 Vgl. Fn. 7 .

39 Eine Vorstellung, von der der Richter nicht lassen konnte, obwohl in diesem spezifischen Fall bereits ausführlich geklärt war, dass die drei Liebenden gar nicht zu dritt miteinander schliefen.

40 Buckel (Fn. 6), 243.

4I Vgl. Buckel (Fn. 6), 248.

42 Vgl. Fn. 37. 
des Rechts. 43 Dass April Divilbiss nicht in Berufung ging, sondern sich trotz der

Unterstützung des Loving More Magazine in das Urteil fügte, mag verschiedene Gründe haben und erfordert tiefer gehende Analysen. Elizabeth F. Emens deutet an, dass es nicht um Geld gegangen sei, sondern dass sie so eingeschüchtert war, dass sie ihre Tochter aufgab und der >biologischen $<$ Großmutter überließ.44 Möglicherweise spielt hier auch die von Aviram konstatierte Skepsis gegenüber dem Recht als sinnvollem Instrument zur Etablierung einer Lebensweise mit hinein. ${ }^{5}$ Die erzieherische, produktive Wirkung des Verfahrens und der Gerichtsentscheidung auf die junge Mutter - und in der Verlängerung auf diejenigen, die einen >Stonewalk-Fall erwarteten und enttäuscht wurden - tritt in dem Fall sehr deutlich zutage.

Die Entscheidung des Juvenile Court in Memphis ist so als Ergebnis eines juridischen Transformationsprozesses innerhalb eines bestimmten gesellschaftlichhistorischen Kräfteverhältnisses zu verstehen, der bestimmte Alltagspraxen und Beziehungsweisen produziert.

\section{Ausblick: See Bebind the Veiled Contracts!}

Das produktive Zusammenspiel von Recht und Beziehungsweisen zeigt, dass hegemoniale Beziehungsweisen in juridischen Verfahren widersprüchlich prozessiert werden. Aus der Perspektive der Poly-Beziehungsweisen stellt sich das Recht als Möglichkeit zur Durchsetzung eigener Interessen eher schwach und regulativ dar und wird skeptisch-distanziert abgelehnt oder ausgespart. Am Fall von April Divilbiss wird zudem deutlich, wie die juridische Regulierung der Intimität normierende Effekte zeitigt. Vor diesen Ergebnissen stellt sich die Frage, ob ein aktiver Umgang mit dem Recht aus der Perspektive nonkonfomer Beziehungsweisen überhaupt möglich ist?

Die Rechts-Skepsis der relativ jungen Polyamory-Bewegung lässt sich in ihrem kulturellen Entstehungskontext verorten, ${ }^{46}$ gleichzeitig sind weitere Entwicklungen an dieser Front nicht abzusehen. Um die Kritik an der herrschenden Zweierbeziehungsnorm auch ins Recht einschreiben zu können, bedarf es eines umfassenden Aufklärungsprojektes, welches gleichermaßen auf die öffentliche Etablierung der Lebensform wie auf die individuell-emotionale Lebbarkeit jener Lebensentwürfe zielt. Ein Ansatz zur kritischen Reflexion monogamer Beziehungsweisen liegt darin, die impliziten Verträge sichtbar zu machen, die allen Beziehungen - ganz gleich, ob exklusiven oder offenen Zweier- oder Mehrfachbeziehungen - als Erwartungen inhärent sind. 47 Recht ist dann nicht mehr als regulative Sanktionsmacht zu verstehen, sondern als produktive, Hegemonie produzierende Form, die über die materielle Stütze juristischer Institutionen hinaus (auch: emotional) durchschlagende Effekte zeitigt. Diese Effekte, diese juridischen Gespenster, sind im Rahmen einer emanzipatorischen Diskussion im Alltag und in der Beziehungspraxis aufzuspüren - ein Ausblenden respektive Verleugnen der Quasi-Vertraglichkeit von Beziehungsarrangements konserviert das

43 Vgl. Antonio Gramsci, Gefängnishefte. Kritische Gesamtausgabe, hrsg. von Klaus Borchmann/Wolfgang Fritz Haug, Hamburg/Berlin I999, H. 6, $\$ 84,777$.

44 Emens (Fn. 30), 3 I 2.

45 Aviram (Fn. 3I).

46 Aviram (Fn. 3I), $23 \mathrm{ff}$.

47 Einen radikalen praktischen Vorschlag dazu macht z.B. die queerfeministische Philosophin Beatriz Preciado, die kontrasexuelle Verträge fordert, welche das Ungesagte in Beziehungen explizit widerrufen. Vgl. Beatriz Preciado, Kontrasexuelles Manifest, Berlin 2003. 
schlechte Bestehende. Anzuknüpfen ist in der Diskussion der Verflechtung der Zweierbeziehungsnorm mit dem Recht an das permanente Scheitern der impliziten Beziehungsverträge, die mannigfaltige Möglichkeiten und widerständige Wünsche ${ }^{4}$ aufscheinen lassen. Diese Wünsche - verstanden als szenische und widersprüchliche Verkörperung des Sozialen - sind hier zur Grundlage einer Politik des Poly-Begehrens zu machen, welche sich in den Hegemonieapparat Recht einzuschreiben vermag.

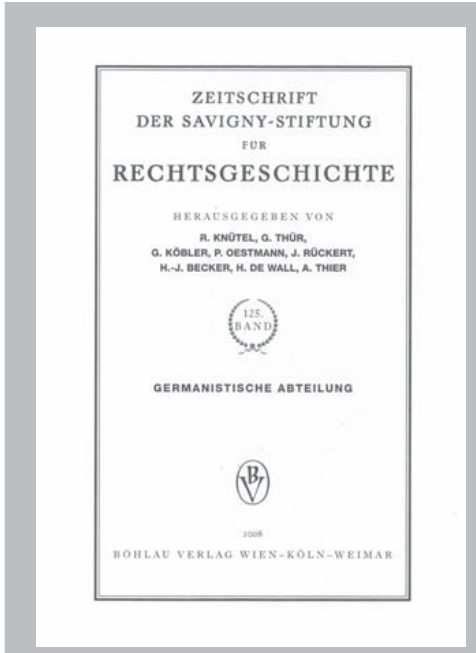

Die Herausgeber des 125. Bandes 2008:

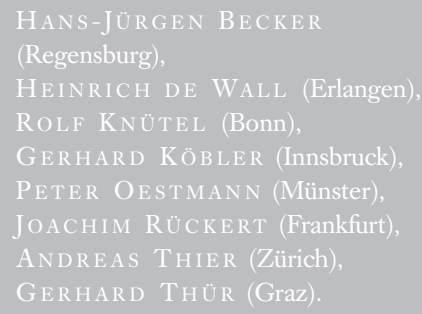

\section{Zeitschrift \\ der Savigny-Stiftung \\ fur Rechtsgeschichte \\ 2008 / 125. Band}

Germanistische Abteilung

ISSN: 0323-4045

XLVIII, 1090 Seiten. Br.

\section{Romanistische Abteilung \\ ISSN: 0323-4096 \\ X, 1035 Seiten. Br.}

Kanonistische Abteilung

ISSN: 0323-4142

VI, 379 Seiten. Br.

\section{8 - Die \\ Zeitschrift \\ der Savigny-Stiftung für Rechtsgeschichte}

\section{erscheint zum 125. Mal}

Der erste Vorgänger der heute vorliegenden ZRG wurde im Jahr 1815 von Friedrich Karl von Savigny und F. Eichhorn im Geiste der Historischen Rechtsschule ins Leben gerufen und gefördert. Erst seit 1861 erscheint „das alte Flaggschiff der deutschsprachigen Rechtsgeschichte“ mehr oder weniger kontinuierlich bis heute - im Jahr 2008 zum 125. Mal!

Eine engere Verbindung mit der SavignyStiftung - deren Namen die ZRG trägt, obwohl die Stiftung ruht - ermöglichte 1880 die vertraute Zweiteilung von Romanistischer und Germanistischer Abteilung. 1911 kam eine eigene Kanonistische Abteilung hinzu. Seither erscheint die ZRG einmal jährlich in den drei unabhängigen Abteilungen Romanistische, Germanistische und Kanonistische Abteilung.

Heute stellt die ZRG einen festen Bestandteil in der europäischen rechtshistorischen Forschung dar. Der Aufsatzteil mit grundsätzlich unveröffentlichten Beiträgen über neu entdeckte Quellenfunde, neue Wertungen von Bekanntem oder vergleichende Beobachtungen prägt maßgeblich den aktuellen Stand der Disziplin. Der Literaturteil berichtet nach Möglichkeit umfassend von den einschlägigen Neuerscheinungen am internationalen Medienmarkt. Chronik und Mitteilungen ergänzen das Angebot. Die ZRG erscheint mit Beiträgen in fünf westeuropäischen Sprachen mit Einschüben in alten Sprachen.

Die nach je 25 Bänden erscheinenden Generalregister der einzelnen Abteilungen erleichtern mit Sach-, Quellen- und Personenverzeichnissen den Zugang zu den Themen.

\section{Böhlau Verlag GmbH \& Co KG} Wien Köln Weimar

Wiesingerstraße 1, 1010 Wien, Österreich.

Telefon (+43 1) $3302427-0$

Telefax (+43 1) 3302432

www.boehlau.at, vertrieb@boehlau.at 